\title{
Trastornos del Ritmo Cardíaco y sospecha de Síndrome de Apneas-Hipopneas del Sueño
}

\author{
Alicia Díaz Baqueroa, Ángeles Sánchez Armengola, \\ Candela Caballero Eraso ${ }^{a}$, Rosa M. Luz Romero ${ }^{a}$
}

\begin{abstract}
a Unidad de Trastornos
Respiratorios del Sueño,

Unidad Médico-Quirúrgica de Enfermedades Respiratorias, Hospital Universitario Virgen del Rocío, Sevilla.

\section{Correspondencia: Ángeles \\ Sánchez-Armengol, Urbanización Ciudad Expo, c/ La Pinta, $n^{\circ} 58,2^{\circ} B$, Mairena del Aljarafe, 41927-Sevilla, España. Telf.: 955012191, fax: 955012191, e-mail: aajm4rs023@ono.com, m.sanchez.armengol.sspa@ juntadeandalucia.es}

Recibido el 12 de enero de 2011.

Aceptado para su publicación el 30 de marzo de 2011.

\section{RESUMEN}

El Síndrome de Apneas-Hipopneas del Sueño (SAHS) está relacionado con diferentes enfermedades cardiovasculares y supone un factor de riesgo para los trastornos del ritmo cardíaco. Recientemente se ha descrito una relación directa temporal entre los eventos respiratorios obstructivos y la aparición de taquicardia ventricular no sostenida (TVNS) y fibrilación auricular (FA) paroxística durante el sueño.

Palabras Clave. Síndromes de la Apnea del Sueño, Arritmias Cardiacas.

\section{ABSTRACT}

Heart Rhythm disorders and suspected Sleep Apnea-Hypopnea syndrome

Sleep Apnea-Hypopnea Syndrome (SAHS) is associated with several cardiovascular diseases and is a risk factor for heart rhythm disorders. A direct temporal relationship between obstructive respiratory events and the onset of non-sustained ventricular tachycardia (NSVT) and paroxysmal atrial fibrillation (AF), during sleep has been reported recently. Key words. Apnea Sleep Syndromes, Arrhythmias, Cardiac.

\section{INTRODUCCIÓN}

EI SAHS ha sido relacionado con diversas enfermedades cardiovasculares, principalmente hipertensión arterial (HTA) y enfermedad cerebrovascular. Además, supone un factor de riesgo para los trastornos del ritmo cardíaco, sobre todo pausas sinusales, TVNS, FA paroxística, extrasístoles ventriculares y bloqueo aurículo-ventricular. Aunque sus mecanismos fisiopatológicos no son bien conocidos, recientemente se ha descrito una relación directa temporal entre los eventos respiratorios obstructivos y la aparición de TVNS y FA paroxística durante el sueño'.

\section{OBSERVACIONES CLÍNICAS}

Varón de 54 años, sin antecedentes familiares de cardiopatía ni muerte súbita, fumador hasta los 34 años, bebedor habitual de 9 copas de alcohol/día, diagnosticado de obesidad e hipertensión arterial. Presentaba episodios autolimitados de palpitaciones, acompañados de mareo y disnea, detectándose el día del ingreso una taquicardia ventricular no sostenida (TVNS) con QRS ancho y morfología de bloqueo de rama izquierda. Ingresó en el hospital con los siguientes hallazgos: obesidad mórbida, apnea, presión arterial $140 / 80 \mathrm{mmHg}$, tonos cardiacos rítmicos sin soplos, analítica de sangre normal y electrocardiograma con ritmo sinusal a $60 \mathrm{lpm}$, complejo QRS estrecho, muesca terminal en el complejo QRS, onda T negativa en V1, segmento PR normal y segmento QTc normal.

Se realizó el siguiente protocolo:

- Ecografía: ventrículo izquierdo no dilatado ni hipertrófico, con fracción de eyección conservada, sin hipoquinesia y válvulas normales.

- Ergometría: sin alteraciones del segmento ST sugestivas de isquemia. 
Respuesta cronotropa adecuada. El esfuerzo desencadenó una TVNS de QRS ancho (figura 1).

- ECG de señal promediada patológico en los tres parámetros analizados.

- Coronariografía: arterias coronarias normales.

- Se indicó una Resonancia magnética nuclear cardíaca, que fue imposible realizar por problemas técnicos, realizándose una ventriculografía (VI de tamaño y función normales, con VD dilatado y FE ligeramente deprimida).

Durante el ingreso permaneció monitorizado, sin presentar en vigilia ningún evento arrítmico. Durante el sueño se detectaron pausas sinusales de 5 a 7 segundos de duración, asociadas a ronquidos y apneas. Se interrogó sobre la existencia de síntomas de síndrome de apneas-hipopneas del sueño (SAHS), refiriendo ser roncador habitual, con apneas observadas durante el sueño y somnolencia excesiva diurna. Debido a la urgencia de corregir las pausas sinusales y a la dificultad para hacer el estudio de sueño de forma inmediata, se decidió instaurar tratamiento empírico con dispositivo de presión positiva continua en la vía aérea (CPAP) automático, con una presión terapéutica de $9 \mathrm{~cm}$ $\mathrm{H}_{2} \mathrm{O}$, con lo que el paciente dejó de roncar y tener apneas durante el sueño, desapareciendo también las pausas sinusales en la telemetría. Al final del ingreso se realizó un estudio electrofisiológico que resultó normal.

El paciente fue dado de alta con tratamiento con CPAP domiciliaria. El diagnóstico de SAHS no pudo confirmarse mediante estudio de sueño por la imposibilidad de deprivación previa de la CPAP. Se le realizó un registro de oximetría nocturna con la CPAP, con un perfil patológico de saturaciones "en dientes de sierra", típico de SAHS (figura 2). Se llevó a cabo una polisomnografía nocturna para titulación de la CPAP (presión óptima de $14 \mathrm{~cm} \mathrm{H}_{2} \mathrm{O}$ ). El paciente ha continuado con tratamiento con CPAP a $14 \mathrm{~cm} \mathrm{H}_{2} \mathrm{O}$, permaneciendo completamente asintomático respecto al SAHS. En las sucesivas revisiones por parte de Cardiología, todos los estudios y controles han sido normales, sin detectarse más eventos arritmogénicos.

\section{COMENTARIOS}

La relación entre el SAHS y diversas enfermedades cardiovasculares ha sido demostrada en estudios poblaciones amplios ${ }^{2,3}$ y actualmente está aceptada la relación causal entre SAHS e HTA, y su asociación con la enfermedad cerebrovascular y la cardiopatía isquémica. Los trastornos del ritmo cardíaco son también más frecuentes en pacientes con SAHS, sobre todo TVNS, $\mathrm{FA}^{4}$, pausas sinusales, bloqueos auriculares y extrasístoles ventriculares. Este paciente presentaba dos de estos tipos de eventos, pausas sinusales y episodios de TVNS, los cuales se presentaban tanto en reposo como con el esfuerzo (con RUN de TVNS en la ergometría, que hubo de detener por exacerbación de los episodios con la actividad adrenérgica), lo que podría estar en relación con la hiperestimulación simpática crónica a la que se ha atribuido dicha arritmia. Los eventos respiratorios obstructivos inducen hipoxemia e hipercapnia intermitente y repetida durante el sueño, alterando la respuesta hemodinámica y autónoma durante el mismo y también se han implicado mecanismos de tipo central y varios reflejos (de origen pulmonar, cardíaco, de barorreceptores arteriales o quimiorreceptores sanguíneos) que desencadenan una activación simpática progresiva durante las apneas ${ }^{5}$.

Por otro lado, las pausas sinusales durante el sueño que se objetivaron en este paciente se describen hasta en el $10 \%$ de los pacientes con $\mathrm{SAHS}^{6,7}$ y se han asociado a un mecanismo funcional de estimulación vagal secundario a la hipoxia, por lo que los estudios electrofisiológicos realizados durante vigilia suelen ser normales, como sucedió también en este caso. En este sentido, la intensidad de las alteraciones oximétricas debidas al SAHS, aunque no se han podido cuantificar basalmente por no haberse realizado un estudio de sueño diagnóstico, se pueden suponer por el hecho de que el registro de oximetría continua nocturna realizado con la CPAP seguía presentando un perfil claramente patológico (figura 2), a pesar de que parte del SAHS, si bien no en su totalidad, estaba corregido con la CPAP. Una consideración importante en este caso es el hecho de no disponer de un estudio basal diagnóstico de SAHS, por las razones que se han expuesto, a las que hay que añadir que el hallazgo de pausas sinusales de 5 a 7 segundos de duración puede indicar implantación de marcapasos, por lo que se dio prioridad al aspecto terapéutico antes que al diagnóstico. De todas formas, hay una serie de datos que permiten asegurar el diagnóstico de SAHS en este caso: los síntomas referidos (ronquidos habituales, con apneas durante el sueño y somnolencia excesiva durante el día, de años de evolución), la respuesta clínica de los mismos a la CPAP, el resultado obtenido con el registro de autoCPAP (el paciente requirió durante toda la noche la aplicación de presión positiva, necesidad 
que se confirmó en la polisomnografía convencional nocturna de titulación) y el perfil característico de SAHS "en dientes de sierra" (con un índice de desaturaciones elevado), a pesar de estar en tratamiento con CPAP, si bien en ese momento con una presión subterapéutica.
Por otro lado, el hecho de que, desde que está correctamente tratado con CPAP, todos los estudios realizados en Cardiología hayan sido normales, sin detectarse más eventos arritmogénicos, parece apuntar a una relación causal entre las arritmias de este paciente y el SAHS. Hasta ahora no se había

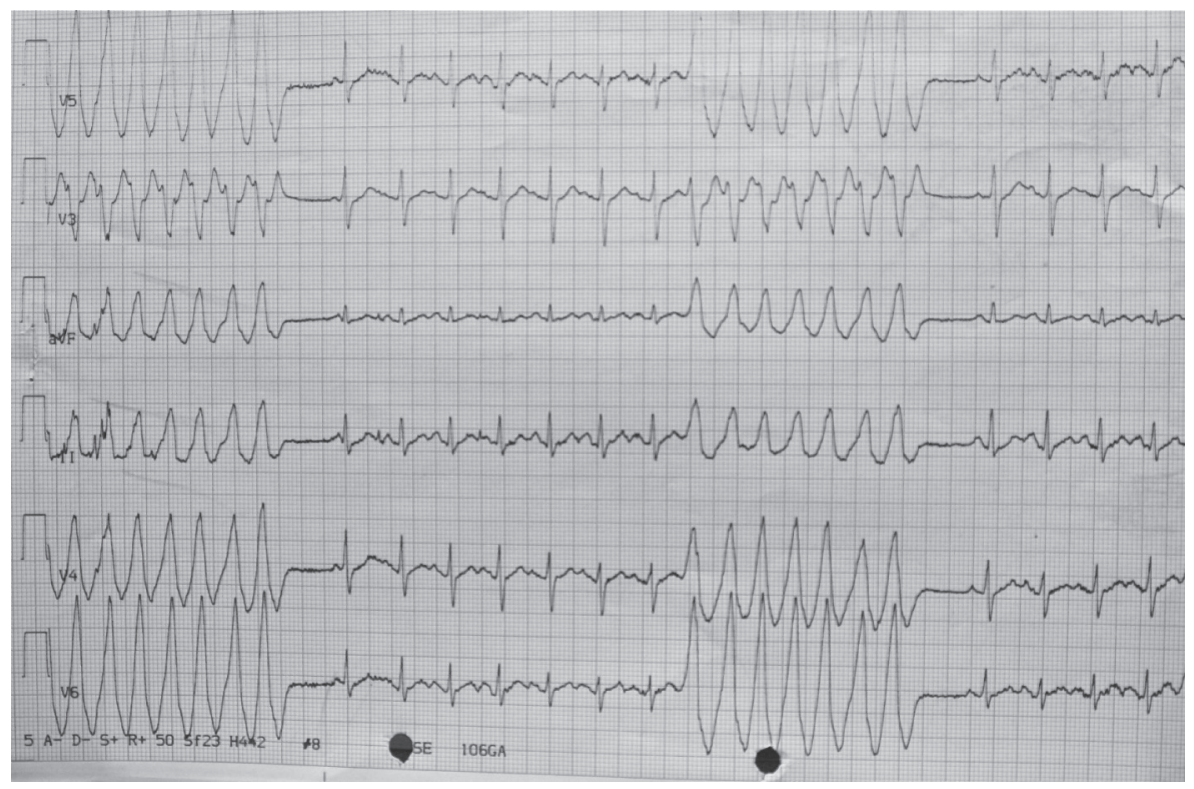

Figura 1. RUN de Taquicardia ventricular no sostenida de QRS ancho (desencadenada por el esfuerzo en la ergometría).

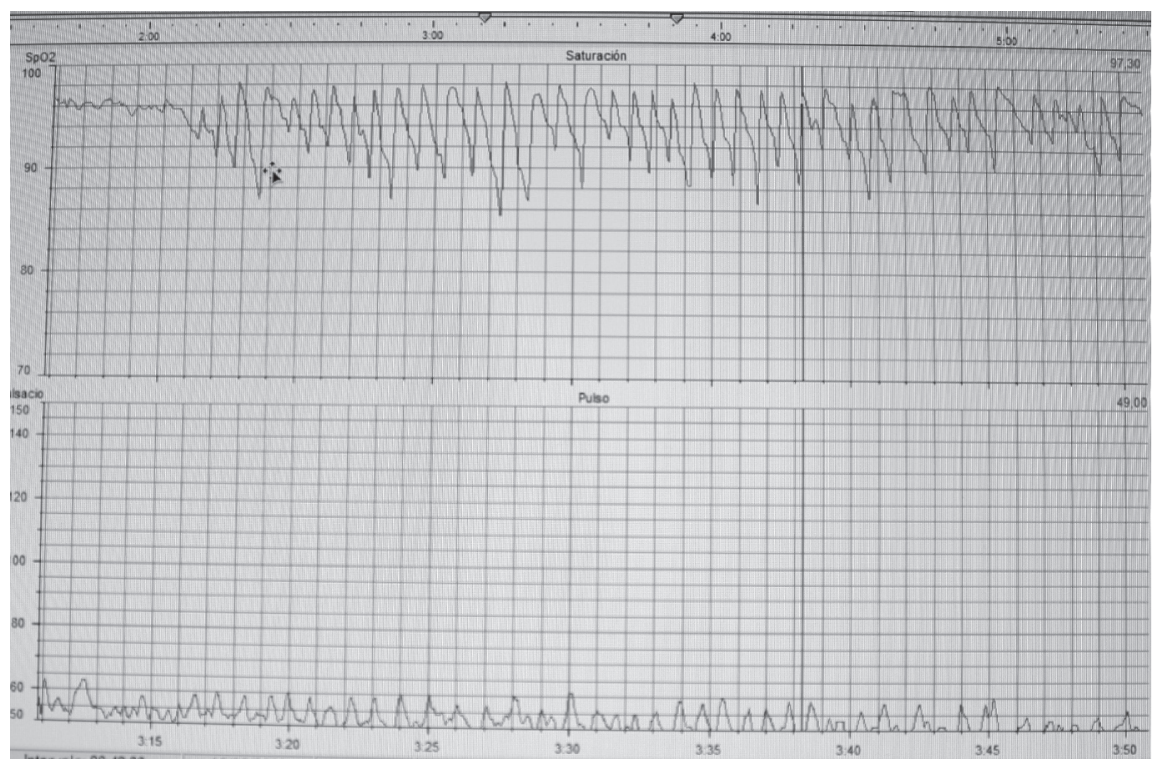

Figura 2. Registro de oximetría nocturna realizado con CPAP a $9 \mathrm{cmH}_{2} \mathrm{O}$, con patrón típico de Síndrome de apneas-hipopneas del sueño (desaturaciones "en dientes de sierra"). 
descrito de forma definitiva una relación causal entre ambas entidades, pero recientemente Monahan et al han evaluado el papel de los trastornos respiratorios del sueño como desencadenantes de eventos arrítmicos nocturnos ${ }^{1}$, encontrando que la TVNS fue la arritmia más frecuente, así como que la incidencia de las alteraciones del ritmo cardíaco fue más elevada en pacientes con alteraciones oximétricas más importantes, todo lo cual es concordante con los hallazgos descritos en este paciente.

\section{BIBLIOGRAFÍA}

1. Monahan K, Storfer-Isser A, Mehra R, Shahar E, Mittleman $\mathrm{M}$, Rottman $\mathrm{J}$ et al. Triggering of nocturnal arrhythmias by sleep-disordered breathing events. J Am Coll Cardiol. 2009; 54(19):1797-804
2. Quan SF, Howard BV, Iber C, Kiley JP, Nieto FJ et al. The Sleep Heart Health Study: design, rationale, and methods. Sleep. 1997; 20(12):1077-85.

3. Peppard EP, Young T, Palta M, Skatrud J. Prospective Study of the Association between Sleep-Disordered Breathing and Hypertension. N Engl J Med. 2000; 342:1378-84.

4. Gami AS, Hodge DO, Herges RM, Olson EJ, Nykodym J, Kara T et al. Obstructive sleep apnea, obesity, and the risk of incident atrial fibrillation. J Am Coll Cardiol. 2007; 49(5):565-71.

5. Leung RS. Sleep-Disordered Breathing: Autonomic Mechanisms and Arrythmias. Prog Cardiovasc Dis. 2009; 51(4):324-38

6. Guilleminault C, Connolly SJ, Winkle RA. Cardiac arrhythmia and conduction disturbance during sleep in 400 patients with sleep apnea syndrome. Am J Cardiol. 1983; 52:490-4

7. Koehler U, Fus E, Grimm W, Pankow W. Schafer H, Stammnitz A et al. Heart block in patients with obstructive sleep apnoea: pathogenetic factors and effects of treatment. Eur Respir J. 1998; 11:434-9. 